Article

\title{
Fuzzy Reduced Hypergroups
}

\author{
Milica Kankaras ${ }^{1(1)}$ and Irina Cristea ${ }^{2, *(1)}$ \\ 1 Department of Mathematics, University of Montenegro, 81000 Podgorica, Montenegro; milica.k@ucg.ac.me \\ 2 Centre for Information Technologies and Applied Mathematics, University of Nova Gorica, \\ 5000 Nova Gorica, Slovenia \\ * Correspondence: irina.cristea@ung.si or irinacri@yahoo.co.uk; Tel.: +386-0533-15-395
}

Received: 10 January 2020; Accepted: 14 February 2020; Published: 17 February 2020

\begin{abstract}
The fuzzyfication of hypercompositional structures has developed in several directions. In this note we follow one direction and extend the classical concept of reducibility in hypergroups to the fuzzy case. In particular we define and study the fuzzy reduced hypergroups. New fundamental relations are defined on a crisp hypergroup endowed with a fuzzy set, that lead to the concept of fuzzy reduced hypergroup. This is a hypergroup in which the equivalence class of any element, with respect to a determined fuzzy set, is a singleton. The most well known fuzzy set considered on a hypergroup is the grade fuzzy set, used for the study of the fuzzy grade of a hypergroup. Based on this, in the second part of the paper, we study the fuzzy reducibility of some particular classes of crisp hypergroups with respect to the grade fuzzy set.
\end{abstract}

Keywords: fuzzy set; reducibility; grade fuzzy set

\section{Introduction}

In the algebraic hypercompositional structures theory, the most natural link with the classical algebraic structures theory is assured by certain equivalences, that work as a bridge between both theories. More explicitly, the quotient structure modulo the equivalence $\beta$ defined on a hypergroup is always a group [1], the quotient structure modulo the equivalence $\Gamma$ defined on a hyperring is a ring [2], while a commutative semigroup can be obtained factorizing a semihypergroup by the equivalence $\gamma$ [3]. A more completed list of such equivalences is very clearly presented in [4]. All the equivalences having this property, i.e., they are the smallest equivalence relations defined on a hypercompositional structure such that the corresponding quotient (modulo that relation) is a classical structure with the same behaviour, are called fundamental relations, while the associated quotients are called fundamental structures. The study of the fundamental relations represents an important topic of research in Hypercompositional Algebra also nowadays [5-7]. But this is not the unique case when the name "fundamental" is given to an equivalence defined on a hyperstructure. Indeed, there exist three other equivalences, called fundamental by Jantosciak [8], who observed that, unlike what happens in classical algebraic structures, two elements can play interchangeable roles with respect to a hyperoperation. In other words, the hyperoperation does not distinguish between the action of the given elements. These particular roles have been translated by the help of the following three equivalences. We say that two elements $x$ and $y$ in a hypergroup $(H, o)$ are:

1. operationally equivalent, if their hyperproducts with all elements in $H$ are the same;

2. inseparable, if $x$ belongs to the same hyperproducts as $y$ belongs to;

3. essentially indistinguishable, if they are both operationally equivalent and inseparable. 
Then a hypergroup is called reduced [8] if the equivalence class of any element with respect to the essentially indistinguishable relation is a singleton. Besides the associated quotient structure (with respect to the same fundamental relation) is a reduced hypergroup, called the reduced form of the original hypergroup. Jantosciak explained the role of these fundamental relations by a very simple and well known result. Define on the set $H=\mathbb{Z} \times \mathbb{Z}^{*}$, where $\mathbb{Z}$ is the set of integers and $\mathbb{Z}^{*}=\mathbb{Z} \backslash\{0\}$, the equivalence $\sim$ that assigns equivalent fractions in the same class: $(x, y) \sim(u, v)$ if and only if $x v=y u$, for $(x, y),(u, v) \in H$. Endow $H$ with a hypercompositional structure, considering the hyperproduct $(w, x) \circ(y, z)=(w z \widehat{+x y}, x z)_{\sim}$, where by $\widehat{x}_{\sim}$ we mean the equivalence class of the element $x$ with respect to the equivalence $\sim$. It follows that the equivalence class of the element $(x, y) \in H$ with respect to all three fundamental relations defined above is equal to the equivalence class of $(x, y)$ with respect to the equivalence $\sim$. Therefore $H$ is not a reduced hypergroup, while its reduced form is isomorphic with $\mathbb{Q}$, the set of rationals.

Motivated by this example, in the same article [8], Jantosciak proposed a method to obtain all hypergroups having a given reduced hypergroup as their reduced form. This aspect of reducibility of hypergroups has been later on considered by Cristea et al. [9-11], obtaining necessary and sufficient condition for a hypergroup associated with a binary or $n$-ary relation to be reduced. The same author studied the regularity aspect of these fundamental relations and started the study of fuzzyfication of the concept of reducibility [12]. This study can be considered in two different directions, corresponding to the two different approaches of the theory of hypergroups associated with fuzzy sets. An overview of this theory is covered by the monograph [13] written by Davvaz and Cristea, the only one on this topic published till now. The fuzzy aspect of reducibility could be investigated in two ways: by studying the indistinguishability between the elements of a fuzzy hypergroup (i.e., a structure endowed with a fuzzy hyperoperation) or by studying the indistinguishability between the images of the elements of a crisp hypergroup through a fuzzy set. By a crisp hypergroup we mean a hypergroup, but the attribute "crisp" is used to emphasize that the structure is not fuzzy. The study conducted in this article follows the second direction, while the first direction will be developed in our future works.

The aim of this note is to study the concept of fuzzy reduced hypergroup as a crisp hypergroup which is fuzzy reduced with respect to the associated fuzzy set. One of the most known fuzzy sets associated with a hypergroup is the grade fuzzy set $\widetilde{\mu}$, introduced by Corsini [14]. It was studied by Corsini and Cristea [15] in order to define the fuzzy grade of a hypergroup as the length of the sequence of join spaces and fuzzy sets associated with the given hypergroup. For any element $x$ in a hypergroup $H$, the value $\widetilde{\mu}(x)$ is defined as the average value of the reciprocals of the sizes of all hyperproducts containing $x$. The properties of this particular fuzzy set, in particular those related to the fuzzy grade, have been investigated for several classes of finite hypergroups, as: complete hypergroups, non-complete 1-hypergroups or i.p.s. hypergroups (i.e., hypergroups with partial scalar identities). Inspired by all these studies, first we introduce the definition of fuzzy reduced hypergroups and present some combinatorial aspects related to them. Then we focus on the fuzzy reducibility of i.p.s. hypergroups, complete hypergroups and non-complete 1-hypergroups with respect to the grade fuzzy set $\tilde{\mu}$. Theorem 3 states that any proper complete hypergroup is not reduced, either fuzzy reduced with respect to $\widetilde{\mu}$. Regarding the i.p.s. hypergroups, we show that they are reduced, but not fuzzy reduced with respect to $\widetilde{\mu}$ (see Theorems 4 and 5). Finally, we present a general method to construct a non-complete 1-hypergroup, that is not reduced either fuzzy reduced with respect to $\tilde{\mu}$. Some conclusions and new research ideas concerning this study are gathered in the last section.

\section{Review of Reduced Hypergroups}

In this section we briefly recall the basic definitions which will be used in the following, as well as the main properties of reduced hypergroups. We start with the three fundamental relations defined by 
Jantosciak [8] on an arbitrary hypergroup. Throughout this note, by a hypergroup $(H, \circ)$, we mean a non-empty set $H$ endowed with a hyperoperation, usually denoted as $\circ: H \times H \rightarrow \mathcal{P}^{*}(H)$ satisfying the associativity, i.e., for all $a, b, c \in H$ there is $(a \circ b) \circ c=a \circ(b \circ c)$ and the reproduction axiom, i.e., for all $a \in H$, there is $a \circ H=H \circ a=H$, where $\mathcal{P}^{*}(H)$ denotes the set of all non-empty subsets of $H$.

Definition 1 ([8]). Two elements $x, y$ in a hypergroup $(H, o)$ are called:

1. operationally equivalent or by short o-equivalent, and write $x \sim_{0} y$, if $x \circ a=y \circ a$, and $a \circ x=a \circ y$, for any $a \in H$;

2. inseparable or by short i-equivalent, and write $x \sim_{i} y$, if, for all $a, b \in H, x \in a \circ b \Longleftrightarrow y \in a \circ b$;

3. essentially indistinguishable or by short e-equivalent, and write $x \sim_{e} y$, if they are operationally equivalent and inseparable.

Definition 2 ([8]). A reduced hypergroup has the equivalence class of any element with respect to the essentially indistinguishable relation $\sim_{e}$ a singleton, i.e., for any $x \in H$, there is $\hat{x}_{e}=\{x\}$.

Example 1. Let $(H, \circ)$ be a hypergroup, where the hyperoperation "o" is defined by the following table.

\begin{tabular}{c|cccc}
$\circ$ & $a$ & $b$ & $c$ & $d$ \\
\hline$a$ & $a$ & $a$ & $a, b, c$ & $a, b, d$ \\
$b$ & $a$ & $a$ & $a, b, c$ & $a, b, d$ \\
$c$ & $a, b, c$ & $a, b, c$ & $a, b, c$ & $c, d$ \\
$d$ & $a, b, d$ & $a, b, d$ & $c, d$ & $a, b, d$
\end{tabular}

One notices that $a \sim_{0} b$, because the lines (and columns) corresponding to $a$ and $b$ are exactly the same, thereby: $\hat{a}_{o}=\hat{b}_{o}=\{a, b\}$, while $\hat{c}_{o}=\{c\}$ and $\hat{d}_{o}=\{d\}$. But, on the other side, the equivalence class of any element in $H$ with respect to the relation $\sim_{i}$ is a singleton, as well as with respect to the relation $\sim_{e}$, by consequence $(H, \circ)$ is reduced.

In [11] Cristea et al. discussed about the regularity of these fundamental relations, proving that in general none of them is strongly regular. This means that the corresponding quotients modulo these equivalences are not classical structures, but hypergroups. Moreover, Jantosciak [8] established the following result.

Theorem 1 ([8]). For any hypergroup $H$, the associated quotient hypergroup $H / \sim_{e}$ is a reduced hypergroup, called the reduced form of $H$.

Motivated by this property, Jantosciak concluded that the study of the hypergroups can be divided into two parts: the study of the reduced hypergroups and the study of all hypergroups having the same reduced form [8].

\section{Fuzzy Reduced Hypergroups}

As already mentioned in the introductory part of this article, the extension of the concept of reducibility to the fuzzy case can be performed on a crisp hypergroup endowed with a fuzzy set, by defining, similarly to the classical case, three equivalences as follows.

Definition 3. Let $(H, \circ)$ be a crisp hypergroup endowed with a fuzzy set $\mu$. For two elements $x, y \in H$, we say that

1. $x$ and $y$ are fuzzy operationally equivalent and write $x \sim_{f o} y$ if, for any $a \in H, \mu(x \circ a)=\mu(y \circ a)$ and $\mu(a \circ x)=\mu(a \circ y)$ 
2. $x$ and $y$ are fuzzy inseparable and write $x \sim_{f i} y$ if $\mu(x) \in \mu(a \circ b) \Longleftrightarrow \mu(y) \in \mu(a \circ b)$, for $a, b \in H$;

3. $x$ and $y$ are fuzzy essentially indistinguishable and write $x \sim_{f e} y$, if they are fuzzy operationally equivalent and fuzzy inseparable.

Definition 4. The crisp hypergroup $(H, \circ)$ is called fuzzy reduced if the equivalence class of any element in $H$ with respect to the fuzzy essentially indistinguishable relation is a singleton, i.e.,

$$
\text { for all } x \in H, \hat{x}_{f e}=\{x\} \text {. }
$$

Notice that the notion of fuzzy reducibility of a hypergroup is strictly connected with the definition of the involved fuzzy set.

Remark 1. It is easy to see that, for each hypergroup $H$ endowed with an arbitrary fuzzy set $\mu$, the following implication holds: for any $a, b \in H$,

$$
a \sim_{o} b \Rightarrow a \sim_{f o} b .
$$

Remark 2. (i) First of all, let us better explain the meaning of $\mu(a \circ b)$, for any $a, b \in H$ and any arbitrary fuzzy set $\mu$ defined on $H$. Generally, $a \circ b$ is a subset of $H$, so $\mu(a \circ b)$ is the direct image of this subset through the fuzzy set $\mu$, i.e., $\mu(a \circ b)=\{\mu(x) \mid x \in a \circ b\}$.

From here, two things need to be stressed on. Firstly, if $a \circ b$ is a singleton, i.e., $a \circ b=\{c\}$, then $\mu(a \circ b)$ is a set containing the real number $\mu(c)$. Therefore we can write $\mu(c) \in \mu(a \circ b)$, but it is not correct writing $\mu(c)=\mu(a \circ b)$, because the first member is a real number, while the second one is a set containing the real number $\mu(c)$.

Secondly, if $a \in x \circ y$, then, clearly $\mu(a) \in \mu(x \circ y)$, but not also viceversa because it could happen that $\mu(a) \in \mu\left(x^{\prime} \circ y^{\prime}\right)$ for $a \notin x^{\prime} \circ y^{\prime}$.

(ii) Generally, $a \sim_{i} b \nRightarrow a \sim_{f i} b$, as illustrated in the next example. Indeed, if $a \sim_{i} b$ then $a \in x \circ y$ if and only if $b \in x \circ y$. But it can happen that $\mu(a) \in \mu\left(x^{\prime} \circ y^{\prime}\right)$ with $a \notin x^{\prime} \circ y^{\prime}$, so also $b \notin x^{\prime} \circ y^{\prime}$. And if $\mu(a) \neq \mu(b)$, then $\mu(b) \notin \mu\left(x^{\prime} \circ y^{\prime}\right)$, thus $a x_{i} b$.

(iii) Finally, it is simple to see the implication $\mu(a)=\mu(b) \Rightarrow a \sim_{f i} b$.

The following example illustrates all the issues in the above mentioned remark.

Example 2 ([16]). Let $(H, O)$ be a hypergroup represented by the following commutative Cayley table:

\begin{tabular}{c|cccc}
$\circ$ & $e$ & $a_{1}$ & $a_{2}$ & $a_{3}$ \\
\hline$e$ & $e$ & $a_{1}$ & $a_{2}, a_{3}$ & $a_{2}, a_{3}$ \\
$a_{1}$ & & $a_{2}, a_{3}$ & $e$ & $e$ \\
$a_{2}$ & & & $a_{1}$ & $a_{1}$ \\
$a_{3}$ & & & & $a_{1}$
\end{tabular}

One notices immediately that $a_{2} \sim_{i} a_{3}$, while $a_{1} \chi_{i} a_{2}$.

(a) Define now on $H$ the fuzzy set $\mu$ as follows: $\mu(e)=1, \mu\left(a_{1}\right)=\mu\left(a_{2}\right)=0.3, \mu\left(a_{3}\right)=0.5$. Since $\mu\left(a_{1}\right)=$ $\mu\left(a_{2}\right)$, it follows that $a_{1} \sim_{f i} a_{2}$. Moreover, since e $\circ a_{1}=\left\{a_{1}\right\}$, we have

$$
\mu\left(e \circ a_{1}\right)=\left\{\mu\left(a_{1}\right)\right\}=\{0.3\} \ni \mu\left(a_{2}\right),
$$

while it is clear that $\mu\left(a_{3}\right) \notin \mu\left(e \circ a_{1}\right)$, so $a_{2} \chi_{f i} a_{3}$. 
(b) If we define on $H$ the fuzzy set $\mu$ by taking $\mu(e)=\mu\left(a_{1}\right)=1, \mu\left(a_{2}\right)=\mu\left(a_{3}\right)=\frac{1}{3}$, it follows that $e \sim_{f i} a_{1}$ and $a_{2} \sim_{f i} a_{3}$.

Once again, it is evident that the three equivalences $\sim_{f o}, \sim_{f i}$, and $\sim_{f e}$ are strictly related with the definition of the fuzzy set considered on the hypergroup.

Now we will present an example of an infinite hypergroup and study its fuzzy reducibility.

Example 3. Consider the partially ordered group $(\mathbb{Z},+, \leq)$ with the usual addition and orderings of integers. Define on $\mathbb{Z}$ the hyperoperation $a * b=\{x \in \mathbb{Z} \mid a+b \leq x\}$. Then $(\mathbb{Z}, *)$ is a hypergroup [17]. Define now on $\mathbb{Z}$ the fuzzy set $\mu$ as follows: $\mu(0)=0$ and $\mu(x)=\frac{1}{|x|}$, for any $x \neq 0$. We obtain $\hat{x}_{\text {f.o. }}=\{x\}$, for any $x \in \mathbb{Z}$, therefore $(\mathbb{Z}, *)$ is fuzzy reduced with respect to $\mu$. Indeed, for two arbitrary elements $x$ and $y$ in $\mathbb{Z}$, we have $x \sim_{f o} y$ if and only if $\mu(x * a)=\mu(y * a)$, for any $a \in \mathbb{Z}$, where $\mu(x * a)=\left\{\frac{1}{|x+a|}, \frac{1}{|x+a+1|}, \frac{1}{|x+a+2|}, \ldots\right\}$ and similarly, $\mu(y * a)=\left\{\frac{1}{|y+a|}, \frac{1}{|y+a+1|}, \ldots\right\}$. Since $a$ is an arbitrary integer, for any $x$ and $y$ we always find a suitable integer a such that $x+a>0$ and $y+a>0$. This means that the sets $\mu(x * a)$ and $\mu(y * a)$ contain descending sequences of positive integers, so they are equal only when $x=y$. Therefore $x \sim_{f o} y$.

In the following, we will study the fuzzy reducibility of some particular types of finite hypergroups, with respect to the grade fuzzy set $\widetilde{\mu}$, defined by Corsini [14]. We recall here its definition. With any crisp hypergroupoid $(H, \circ)$ (not necessarily a hypergroup) we may associate the fuzzy set $\tilde{\mu}$ considering, for any $u \in H$,

$$
\widetilde{\mu}(u)=\frac{\sum_{(x, y) \in Q(u)} \frac{1}{|x \circ y|}}{q(u)},
$$

where $Q(u)=\left\{(a, b) \in H^{2} \mid u \in a \circ b\right\}$ and $q(u)=|Q(u)|$. By convention, we take $\widetilde{\mu}(u)=0$ anytime when $Q(u)=\varnothing$. In other words, the value $\widetilde{\mu}(u)$ is the average value of reciprocals of the sizes of all hyperproducts $x \circ y$ containing the element $u$ in $H$. In addition, sometimes when we will refer to formula (1), we will denote its numerator by $A(u)$, while the denominator is already denoted by $q(u)$.

Remark 3. As already explained in Remark 2 (ii), generally, for an arbitrary fuzzy set, $x \sim_{i} y \not \Rightarrow x \sim_{f i} y$, while the implication holds if we consider the grade fuzzy set $\tilde{\mu}$. Indeed, if $x \sim_{i} y$, then $x \in a \circ b$ if and only if $y \in a \circ b$ and therefore $Q(x)=Q(y)$, implying that $q(x)=q(y)$, and moreover $A(x)=A(y)$. This leads to the equality $\widetilde{\mu}(x)=\widetilde{\mu}(y)$. By consequence, based on Remark 2 (iii), it holds $x \sim_{f i} y$, with respect to $\widetilde{\mu}$.

Example 4. Let us consider now a total finite hypergroup $H$, i.e., $x \circ y=H$, for all $x, y \in H$. It is easy to see that $x \sim_{e} y$ for any $x, y \in H$, meaning that $\hat{x}_{e}=H$, for any $x \in H$. Thus, a total hypergroup is not reduced. What can we say about the fuzzy reducibility with respect to the grade fuzzy set $\widetilde{\mu}$ ?

For any $u \in H$, there is

$$
\widetilde{\mu}(u)=\frac{|H|^{2} \frac{1}{|H|}}{|H|^{2}}=\frac{1}{|H|} .
$$

Since, $x \sim_{0} y$ for any $x, y \in H$, it follows that $x \sim_{f o} y$, for any $x, y \in H$. Then, it is clear that $\widetilde{\mu}(x)=\widetilde{\mu}(y)$, for all $x, y \in H$, implying that $x \sim_{f i} y$, for all $x, y \in H$. Concluding, it follows that any total finite hypergroup is neither reduced, nor fuzzy reduced.

Based now on Remarks 1 and 3, the following assertion is clear. 
Corollary 1. If $(H, \circ)$ is a not reduced hypergoup, then it is also not fuzzy reduced with respect to the grade fuzzy set $\widetilde{\mu}$.

\subsection{Fuzzy Reducibility in Complete Hypergroups}

The complete hypergroups form a particular class of hypergroups, strictly related with the join spaces and the regular hypergroups. Their definition is based on the notion of complete part, introduced in Koskas [1], with the main role to characterize the equivalence class of an element under the relation $\beta^{*}$. More exactly, a nonempty set $A$ of a semihypergroup $(H, \circ)$ is called a complete part of $H$, if for any natural number $n$ and any elements $a_{1}, a_{2}, \ldots, a_{n}$ in $H$, the following implication holds:

$$
A \cap \prod_{i=1}^{n} a_{i} \neq \varnothing \Rightarrow \prod_{i=1}^{n} a_{i} \subseteq A .
$$

We may say, as it was mentioned in the review written by Antampoufis et al. [18], that a complete part $A$ absorbs all hyperproducts of the elements of $H$ having non-empty intersection with $A$. The intersection of all complete parts of $H$ containing the subset $A$ is called the complete closure of $A$ in $H$ and denoted by $C(A)$.

Definition 5. A hypergroup $(H, o)$ is called a complete hypergroup if, for any $x, y \in H$, there is $C(x \circ y)=x \circ y$.

As already explained in the fundamental book on hypergroups theory [19] and the other manuscripts related with complete hypergroups $[16,20]$, in practice, it is more useful to use the following characterization of the complete hypergroups.

Theorem 2 ([19]). Any complete hypergroup may be constructed as the union $H=\bigcup_{g \in G} A_{g}$ of its subsets, where

$(1)(G, \cdot)$ is a group.

(2) The family $\left\{A_{g}, \mid g \in G\right\}$ is a partition of $G$, i.e., for any $\left(g_{1}, g_{2}\right) \in G^{2}, g_{1} \neq g_{2}$, there is $A_{g_{1}} \cap A_{g_{2}}=\varnothing$.

(3) If $(a, b) \in A_{g_{1}} \times A_{g_{2}}$, then $a \circ b=A_{g_{1} g_{2}}$.

Example 5. The hypergroup presented in Example 2 is complete, where the group $G=\left(\mathbb{Z}_{3},+\right)$ and the partition set contains $A_{0}=\{e\}, A_{1}=\left\{a_{1}\right\}$, and $A_{2}=\left\{a_{2}, a_{3}\right\}$. It is clear that all conditions in Theorem 2 are fulfilled.

Let $(H, \circ)$ be a proper complete hypergroup (i.e., $H$ is not a group). Define now on $H$ the equivalence " " by:

$$
x \sim y \Longleftrightarrow \exists g \in G \text { such that } x, y \in A_{g} .
$$

Proposition 1. On a proper complete hypergroup $(H, \circ)$, the equivalence in (2) is a representation of the essentially indistinguishability equivalence $\sim_{e}$.

Proof. By Theorem 2, one notices that, for any element $x \in H$, there exists a unique $g \in G$ such that $x \in A_{g_{x}}$. In the following, we will denote this element by $g_{x}$. First, suppose that $x \sim y$, i.e., there exists $g_{x}=g_{y} \in G$ such that $x, y \in A_{g_{x}}$. For any arbitrary element $a \in H$, we can say that $a \in A_{g_{a}}$, with $g_{a} \in G$, and by the definition of the hyperproduct in the complete hypergroup $(H, \circ)$, there is $x \circ a=A_{g_{x} g_{a}}=A_{g_{y} g_{a}}=y \circ a$, (and similarly, $a \circ x=a \circ y$,) implying that $x \sim_{0} y$ (i.e., $x$ and $y$ are operationally equivalent). Secondly, for any $x \in a \circ b=A_{g_{a} g_{b}} \cap A_{g_{x}}$, it follows that $g_{a} g_{b}=g_{x}$; but $g_{x}=g_{y}$, so $y \in A_{g_{a} g_{b}}=a \circ b$. Thereby, 
$x \in a \circ b$ if and only if $y \in a \circ b$, meaning that $x \sim_{i} y$ (i.e., $x$ and $y$ are inseparable). We have proved that $\sim \subseteq \sim_{e}$.

Conversely, let us suppose that $x \sim_{e} y$. Since $x$ and $y$ are inseparable, i.e., $x \in a \circ b$ if and only if $y \in a \circ b$, we may write $x, y \in A_{g_{a} g_{b}}$. Therefore there exists $g_{x}=g_{a} \cdot g_{b} \in G$ such that $x, y \in A_{g_{x}}$, so $x \sim y$.

Example 6. If we continue with Example 2, we notice that the equivalence classes of the elements of $H$ with respect to the equivalence $\sim$ defined in (2) are: $\hat{e}=\{e\}, \hat{a_{1}}=\left\{a_{1}\right\}, \hat{a_{2}}=\left\{a_{2}, a_{3}\right\}=\hat{a_{3}}$. Regarding now the essentially indistinguishability equivalence $\sim_{e}$, we have the same equivalences classes: $\hat{e}_{e}=\{e\}, \hat{a_{1 e}}=\left\{a_{1}\right\}$, $\hat{a_{2 e}}=\left\{a_{2}, a_{3}\right\}=\hat{a_{3 e}}$. This is clear from the Cayley table of the hypergroup: the lines corresponding to $a_{2}$ and $a_{3}$ are the same and every time $a_{2}$ and $a_{3}$ are in the same hyperproduct, so they are equivalent. Since these properties are not satisfied for $a_{1}$ and e, their equivalence classes are singletons.

Proposition 2. Let $(H, \circ)$ be a proper complete hypergroup and consider on $H$ the grade fuzzy set $\widetilde{\mu}$. Then $\sim \subset \sim$ fe (with respect to the fuzzy set $\widetilde{\mu}$ ).

Proof. By the definition of the grade fuzzy set $\widetilde{\mu}$, one obtains that

$$
\widetilde{\mu}(x)=\frac{1}{\left|A_{g_{x}}\right|} \text {, for any } x \in H \text {. }
$$

Take now $x, y \in H$ such that $x \sim y$. There exists $g_{x} \in G$ such that $x, y \in A_{g_{x}}$, thereby $\widetilde{\mu}(x)=\widetilde{\mu}(y)$ and by Remark 2 (ii) we have $x \sim_{f i} y$. Moreover, by Proposition 1 there is $x \sim_{o} y$ and by Remark 1 we get that $x \sim_{f o} y$. Concluding, we have proved that $x \sim y \Rightarrow x \sim_{f e} y$, with respect to $\widetilde{\mu}$.

Theorem 3. Any proper complete hypergroup is not reduced, either fuzzy reduced with respect to the grade fuzzy set.

Proof. Since $(H, O)$ is a proper complete hypergroup, there exists at least one element $g$ in $G$ such that $\left|A_{g}\right| \geq 2$, i.e., there exist two distinct elements $a$ and $b$ in $H$ with the property that $a \sim b$. Then $a \sim_{e} b$ and $a \sim_{f e} b$, meaning that $(H, \circ)$ is not reduced, either fuzzy reduced with respect to the grade fuzzy set $\widetilde{\mu}$.

\subsection{Fuzzy Reducibility in i.p.s. Hypergroups}

An i.p.s. hypergroup is a canonical hypergroup with partial scalar identities. The name, given by Corsini [21], originally comes from Italian, the abbreviation "i.p.s." standing for "identità parziale scalare" (in English, partial scalar identity). First we dwell on the terminology connected with the notion of identity in a hypergroup $(H, \circ)$. An element $x \in H$ is called a scalar, if $|x \circ y|=|y \circ x|=1$, for any $y \in H$. An element $e \in H$ is called partial identity of $H$ if it is a left identity (i.e., there exists $x \in H$ such that $x \in e \circ x$ ) or a right identity (i.e., there exists $y \in H$ such that $y \in y \circ e$ ). We denote by $I_{p}$ the set of all partial identities of $H$. Besides, for a given element $x \in H$, a partial identity of $x$ is an element $u \in H$ such that $x \in x \circ u \cup u \circ x$. The element $u \in H$ is a partial scalar identity of $x$ whenever from $x \in x \circ u$ it follows $x=x \circ u$ and from $x \in u \circ x$ it follows that $x=u \circ x$. We denote by $I_{p}(x)$ the set of all partial identities of $x$, by $I_{p s}(x)$ the set of all partial scalar identities of $x$, and by $S c(H)$ the set of all scalars of $H$. It is obvious that $I_{p s}(x)=I_{p}(x) \cap S c(H)$.

Remark 4. The term "partial" here must not be confused with the "left or right" (identity), but it must be connected with the fact that the element $u$ has a partial behaviour of identity with respect to the element $x$. So, $u$ is not a left/right (i.e., partial) identity for the hypergroup H. Besides, an i.p.s. hypergroup is a commutative hypergroup, so the concept of partial intended as left/right element satisfying a property (i.e., left/right unit) has no sense. It is 
probably better to understand an element $u$ having the property of being partial identity for $x$ as an element having $a$ similar behaviour as an identity but only with respect to $x$, so a partial role of being identity.

Let us recall now the definition of an i.p.s. hypergroup. All finite i.p.s. hypergroups of order less than 9 have been determined by Corsini [21-23].

Definition 6. A hypergroup $(H, \circ)$ is called i.p.s. hypergroup, if it satisfies the following conditions.

1. It is canonical, i.e.

- it is commutative;

- it has a scalar identity 0 such that $0 \circ x=x$, for any $x \in H$;

- $\quad$ every element $x \in H$ has a unique inverse $x^{-1} \in H$, that is $0 \in x \circ x^{-1}$;

- it is reversible, so $y \in a \circ x \Longrightarrow x \in a^{-1} \circ y$, for any $a, x, y \in H$.

2. It satisfies the relation: for any $a, x \in H$, if $x \in a \circ x$, then $a \circ x=x$.

The most useful properties of i.p.s. hypergroups are gathered in the following result.

Proposition 3 ([21]). Let $(H, \circ)$ be an i.p.s. hypergroup.

1. For any element $x$ in $H$, the set $x \circ x^{-1}$ is a subhypergroup of $H$.

2. For any element $x$ in $H$, different from $\{0\}$, we have: or $x$ is a scalar of $H$, or there exists $u \in S c(H) \backslash\{0\}$ such that $u \in x \circ x^{-1}$. Moreover $|S c(H)| \geq 2$.

3. If $x$ is a scalar of $H$, then the set of all partial scalar identities of $x$ contains just 0.

If $x$ is not a scalar of $H$, then $I_{p s}(x) \subset S c(H) \cap x \circ x^{-1}$ and therefore $\left|I_{p s}(x)\right| \geq 2$.

Proposition 4. Let $(H, O)$ be an i.p.s. hypergroup. For any scalar $u \in H$ and for any element $x \in H$, there exists a unique $y \in H$ such that $u \in x \circ y$.

Proof. The existence part immediately follows from the reproducibility property of $H$. For proving the unicity, assume that there exist $y_{1}, y_{2} \in H, y_{1} \neq y_{2}$ such that $u \in x \circ y_{1} \cap x \circ y_{2}$. Then, by reversibility, it follows that $y_{1}, y_{2} \in x^{-1} \circ u$. Since $u$ is a scalar element, we get $\left|x^{-1} \circ u\right|=1$ and then $y_{1}=y_{2}=$ $x^{-1} \circ u$.

Example 7 ([21]). Let us consider the following i.p.s. hypergroup $(H, \circ)$.

\begin{tabular}{c|cccc}
$H$ & 0 & 1 & 2 & 3 \\
\hline 0 & 0 & 1 & 2 & 3 \\
1 & 1 & 2 & 0,3 & 1 \\
2 & 2 & 0,3 & 1 & 2 \\
3 & 3 & 1 & 2 & 0
\end{tabular}

Here we can notice that 0 is the only one identity of $H$. In addition, $S c(H)=\{0,3\}$, and $0 \in 0 \circ u$ only for $u=0$, so $I_{p s}(0)=\{0\}$. Also, only for $u=0$ there is $3 \in 3 \circ u$, thus $I_{p s}(3)=\{0\}$. (In general, if $x \in S c(H)$ then $I_{p s}(x)=\{0\}$, according with Proposition 3). Similarly, one gets $I_{p}(1)=I_{p}(2)=\{0,3\}$ and since $S c(H)=\{0,3\}$, it follows that $I_{p s}(1)=I_{p s}(2)=\{0,3\}$. 
Note that, in an i.p.s. hypergroup, the Jantosciak fundamental relations have a particular meaning, in the sense that, for any two elements there is

$$
a \sim_{o} b \Longleftrightarrow a \sim_{i} b \Longleftrightarrow a \sim_{e} b \Longleftrightarrow a=b
$$

By consequence, one obtains the following result.

Theorem 4. Any i.p.s. hypergroup is reduced.

In the following we will discuss the fuzzy reducibility of an i.p.s. hypergroup with respect to the grade fuzzy set $\tilde{\mu}$.

Theorem 5. Any i.p.s. hypergroup is not fuzzy reduced with respect to the fuzzy set $\tilde{\mu}$.

Proof. Since any i.p.s. hypergroup contains at least one non-zero scalar, take arbitrary such a $u \in S c(H)$. We will prove that $u \sim_{f i} 0$ and $u \sim_{f o} 0$, therefore $\left|\hat{0}_{f e}\right| \geq 2$, meaning that $H$ is not fuzzy reduced.

First we will prove that, for any $u \in S c(H)$, there is $\widetilde{\mu}(0)=\widetilde{\mu}(u)$, equivalently with $u \sim_{f i} 0$. For doing this, based on the fact that $\widetilde{\mu}(x)=\frac{A(x)}{q(x)}$, for all $x \in H$, we show that $A(0)=A(u)$ and $q(0)=q(u)$.

Let us start with the computation of $q(0)$ and $q(u)$. If $0 \in x \circ y$, it follows that $y \in x^{-1} \circ 0$, that is $y=$ $x^{-1}$ and then $Q(0)=\left\{(x, y) \in H^{2} \mid 0 \in x \circ y\right\}=\left\{\left(x, x^{-1}\right) \mid x \in H\right\}$. Thereby $q(0)=|Q(0)|=n=|H|$. On the other hand, by Proposition 4, we have $q(u)=n=|H|$ (since for any $x \in H$ there exists a unique $y \in H$ such that $u \in x \circ y$.)

Let us calculate now $A(0)$. By formula (1), we get that

$$
\begin{gathered}
A(0)=\sum_{(x, y) \in Q(0)} \frac{1}{|x \circ y|}=\sum_{x \in H} \frac{1}{\left|x \circ x^{-1}\right|}= \\
\sum_{a \in S c(H)} \frac{1}{\left|a \circ a^{-1}\right|}+\sum_{x \notin S c(H)} \frac{1}{\left|x \circ x^{-1}\right|}=|S c(H)|+\sum_{x \notin S c(H)} \frac{1}{\left|x \circ x^{-1}\right|} .
\end{gathered}
$$

Since, for $u \in S c(H), \exists x \notin S c(H)$ such that $u \in x \circ x^{-1} \cap I_{p s}(x)$, we similarly get that

$$
A(u)=|S c(H)|+\sum_{x \notin S c(H)} \frac{1}{\left|x \circ x^{-1}\right|}
$$

and it is clear that $A(0)=A(u)$, so $\widetilde{\mu}(0)=\widetilde{\mu}(u)$. Therefore $0 \sim_{f i} u$.

It remains to prove the second part of the theorem, that is $0 \sim_{f o} u$, equivalently with $\tilde{\mu}(0 \circ x)=$ $\widetilde{\mu}(u \circ x), \forall x \in H$.

If $x \in S c(H)$, then $u \circ x \in S c(H)$ and by the first part of the theorem, there is $\widetilde{\mu}(u \circ x)=\widetilde{\mu}(0)=$ $\widetilde{\mu}(x)=\widetilde{\mu}(0 \circ x)$.

If $x \notin S c(H)$, then since $S c(H) \subset I_{p s}(a)$, for any $a \notin S c(H)$, it follows that $S c(H) \subset I_{p s}(x)$, so $u \circ x=$ $x$, and then $\widetilde{\mu}(u \circ x)=\widetilde{\mu}(x)=\widetilde{\mu}(0 \circ x)$. Now the proof is complete.

\subsection{Fuzzy Reducibility in Non-Complete 1-Hypergroups}

In this subsection we will study the reducibility and fuzzy reducibility of some particular finite non-complete 1-hypergroups defined and investigated by Corsini, Cristea [24] with respect to their fuzzy grade. A hypergroup $H$ is called 1-hypergroup if the cardinality of its heart $\omega_{H}$ is 1 . 
The general construction of this particular hypergroup is the following one. Consider the set $H=$ $H_{n}=\{e\} \cup A \cup B$, where $A=\left\{a_{1}, \ldots, a_{\alpha}\right\}$ and $B=\left\{b_{1}, \ldots, b_{\beta}\right\}$, with $\alpha, \beta \geq 2$ and $n=\alpha+\beta+1$, such that $A \cap B=\varnothing$ and $e \notin A \cup B$. Define on $H$ the hyperoperation " $\circ$ " by the following rule:

- for all $a \in A, a \circ a=b_{1}$,

- for all $\left(a_{1}, a_{2}\right) \in A^{2}$ such that $a_{1} \neq a_{2}$, set $a_{1} \circ a_{2}=B$,

- for all $(a, b) \in A \times B$, set $a \circ b=b \circ a=e$,

- for all $\left(b, b^{\prime}\right) \in B^{2}$, there is $b \circ b^{\prime}=A$,

- for all $a \in A$, set $a \circ e=e \circ a=A$,

- for all $b \in B, b \circ e=e \circ b=B$ and

- $e \circ e=e$.

$H_{n}$ is an 1-hypergroup which is not complete.

We will discuss the (fuzzy) reducibility of this hypergroup for different cardinalities of the sets $A$ and $B$.

(1) Let us suppose $n=\left|H_{6}\right|=6$, where $H=H_{6}=e \cup A \cup B, \alpha=|A|=2, \beta=|B|=3, A \cap B=\varnothing$, $e \notin A \cup B$ with $A=\left\{a_{1}, a_{2}\right\}, B=\left\{b_{1}, b_{2}, b_{3}\right\}$. Thus the Cayley table of $\left(H_{6}, 0\right)$ is the following one

\begin{tabular}{c|cccccc}
$H$ & $e$ & $a_{1}$ & $a_{2}$ & $b_{1}$ & $b_{2}$ & $b_{3}$ \\
\hline$e$ & $e$ & $A$ & $A$ & $B$ & $B$ & $B$ \\
$a_{1}$ & & $b_{1}$ & $B$ & $e$ & $e$ & $e$ \\
$a_{2}$ & & & $b_{1}$ & $e$ & $e$ & $e$ \\
$b_{1}$ & & & & $A$ & $A$ & $A$ \\
$b_{2}$ & & & & & $A$ & $A$ \\
$b_{3}$ & & & & & & $A$
\end{tabular}

From the table, we notice immediately that the elements $b_{2}$ and $b_{3}$ are essentially indistinguishable, while the equivalence class with respect to the $\sim_{e}$ relation of all other elements is a singleton. Thereby $H$ is not reduced.

Calculating now the values of the grade fuzzy set $\widetilde{\mu}$, one obtains $\widetilde{\mu}(e)=1, \widetilde{\mu}\left(a_{1}\right)=\widetilde{\mu}\left(a_{2}\right)=$ $0.5, \widetilde{\mu}\left(b_{1}\right)=0.467, \widetilde{\mu}\left(b_{2}\right)=\widetilde{\mu}\left(b_{3}\right)=0.333$. Since $\widetilde{\mu}\left(a_{1}\right)=\widetilde{\mu}\left(a_{2}\right)$, it follows that $a_{1} \sim_{f i} a_{2}$. But $\widetilde{\mu}\left(a_{1} \circ a_{1}\right)=$ $\widetilde{\mu}\left(\left\{b_{1}\right\}\right)=\left\{\widetilde{\mu}\left(b_{1}\right)\right\}$, while $\widetilde{\mu}\left(a_{1} \circ a_{2}\right)=\widetilde{\mu}(B)=\left\{\widetilde{\mu}\left(b_{1}\right), \widetilde{\mu}\left(b_{2}\right)\right\}$, so $\widetilde{\mu}\left(a_{1} \circ a_{1}\right) \neq \widetilde{\mu}\left(a_{1} \circ a_{2}\right)$, meaning that $a_{1} \chi_{f o} a_{2}$, that is $a_{1} \chi_{f e} a_{2}$.

On the other side, we have $b_{2} \sim_{f o} b_{3}$ because they are also operationally equivalent, and $b_{2} \sim_{f i} b_{3}$ because $\widetilde{\mu}\left(b_{2}\right)=\widetilde{\mu}\left(b_{3}\right)$. This is equivalent with $b_{2} \sim_{f e} b_{3}$ and therefore $H$ is not reduced with respect to $\widetilde{\mu}$.

(2) Consider now the most general case. The Cayley table of the hypergroup $H$ is the following one:

\begin{tabular}{c|ccccccccc}
$H$ & $e$ & $a_{1}$ & $a_{2}$ & $\cdots$ & $a_{\alpha}$ & $b_{1}$ & $b_{2}$ & $\cdots$ & $b_{\beta}$ \\
\hline$e$ & $e$ & $A$ & $A$ & $\cdots$ & $A$ & $B$ & $B$ & $\cdots$ & $B$ \\
$a_{1}$ & & $b_{1}$ & $B$ & $\cdots$ & $B$ & $e$ & $e$ & $\cdots$ & $e$ \\
$a_{2}$ & & & $b_{1}$ & $\cdots$ & $B$ & $e$ & $e$ & $\cdots$ & $e$ \\
$\vdots$ & & & & $\ddots$ & & & & & $\vdots$ \\
$a_{\alpha}$ & & & & & $b_{1}$ & $e$ & $e$ & $\cdots$ & $e$ \\
$b_{1}$ & & & & & & $A$ & $A$ & $\cdots$ & $A$ \\
$b_{2}$ & & & & & & & $A$ & $\cdots$ & $A$ \\
$\vdots$ & & & & & & & & $\ddots$ & $\vdots$ \\
$b_{\beta}$ & & & & & & & & & $A$
\end{tabular}


As already calculated in [24], there is $\widetilde{\mu}(e)=1, \widetilde{\mu}\left(a_{i}\right)=\frac{1}{\alpha}$, for any $i=1, \ldots, \alpha$, while $\widetilde{\mu}\left(b_{1}\right)=$ $\frac{\alpha^{2}+\alpha \beta+2 \beta-\alpha}{\beta\left(\alpha^{2}+2 \beta\right)}$, and $\widetilde{\mu}\left(b_{j}\right)=\frac{1}{\beta}$, for any $j=2, \ldots, \beta$. As in the previous case, we see that any two elements in $B \backslash\left\{b_{1}\right\}$ are operational equivalent, by consequence also fuzzy operational equivalent, and indistinguishable and fuzzy indistinguishable (because their values under the grade fuzzy set $\tilde{\mu}$ are the same). Concluding, this non complete 1-hypergroup is always not reduced, either not fuzzy reduced with respect to $\widetilde{\mu}$.

\section{Conclusions and Open Problems}

The pioneering paper of Rosenfeld [25] on fuzzy subgroups has opened a new perspective of the study of algebraic structures (hyperstructures) using the combinatorial properties of fuzzy sets. Regarding this, there are two distinct views: one concerns the crisp (hyper)structures endowed with fuzzy sets, and the other one is related to fuzzy (hyper)structures, that are sets on which fuzzy (hyper)operations are defined. Several classical algebraic concepts have then been extended in the framework of these two lines of research, reducibility in hypergroups, defined by Jantosciak [8] already in 1990, being one of them. In this paper, we have focussed on the study of fuzzy reducibility in hypergroups, extending the concept of reducibility to hypergroups endowed with fuzzy sets. For a better understanding of this subject, we have chosen the grade fuzzy set $\widetilde{\mu}$ [14] and have investigated the fuzzy reducibility of some particular classes of hypergroups: those with scalar partial identities, complete hypergroups, and special non-complete 1-hypergroups. Several connections between reducibility and fuzzy reducibility (with respect to $\widetilde{\mu}$ ) have been underlined and motivated by several examples.

In our future work, following the other connection between fuzzy sets and (hyper)structures, we aim to describe the reducibility of fuzzy hypergroups [26], by studying the reduced fuzzy hypergroups: they are fuzzy hypergroups which are reduced. Moreover, these aspects will also be investigated in the case of the mimic fuzzy hypergroups [27].

Author Contributions: Conceptualization, M.K. and I.C.; methodology, M.K. and I.C.; investigation, M.K. and I.C.; writing-original draft preparation, M.K.; writing—review and editing, I.C.; supervision, I.C.; funding acquisition, I.C. All authors have read and agreed to the published version of the manuscript.

Funding: The second author acknowledges the financial support from the Slovenian Research Agency (research core funding No. P1-0285).

Conflicts of Interest: The authors declare no conflict of interest.

\section{References}

1. Koskas, M. Groupoides, demi-hypergroupes et hypergroupes. J. Math. Pure Appl. 1970, 49, 155-192.

2. Vougiouklis, T. The Fundamental Relation in Hyperrings. the General Hyperfield. In Algebraic Hyperstructures and Applications (Xanthi, 1990); World Sci. Publishing: Teaneck, NJ, USA, 1991; pp. 203-211.

3. Freni, D. A new characterization of the derived hypergroup via strongly regular equivalences. Commun. Algebra 2002, 30, 3977-3989. [CrossRef]

4. Norouzi, M.; Cristea, I. Fundamental relation on m-idempotent hyperrings. J. Open Math. 2017, 15, 1558-1567. [CrossRef]

5. De Salvo, M.; Fasino, D.; Freni, D.; Lo Faro, G. On hypergroups with a $\beta$-class of finite height. Symmetry 2020, 12, 168. [CrossRef]

6. Zadeh, A.; Norouzi, M.; Cristea, I. The commutative quotient structure of m-idempotent hyperrings. An. Stiintifice Univ. Ovidius Constanta Ser. Mat. 2020, 28, 219-236.

7. Ameri, R.; Eyvazi, M.; Hoskova-Mayerova, S. Superring of Polynomials over a Hyperring. Mathematics 2019, 7, 902. [CrossRef] 
8. Jantosciak, J. Reduced hypergroups. In Proceedings of the Algebraic Hyperstructures and Applications Proceedings of 4th International Congress, Xanthi, Greece, 27-30 June 1990; World Scientific: Singapore, 1991; pp. 119-122.

9. Cristea, I. Several aspects on the hypergroups associated with $n$-ary relations. An. Stiintifice Univ. Ovidius Constanta Ser. Mat. 2009, 17, 99-110.

10. Cristea, I.; Ştefănescu, M. Hypergroups and n-ary relations. Eur. J. Comb. 2010, 31, 780-789. [CrossRef]

11. Cristea, I.; Ştefănescu, M.; Angheluța, C. About the fundamental relations defined on the hypergroupoids associated with binary relations. Eur. J. Comb. 2011, 32, 72-81. [CrossRef]

12. Cristea, I. Reducibility in hypergroups (crisp and fuzzy case). Int. J. Algebr. Hyperstructures Its Appl. 2015, 2, 67-76.

13. Davvaz, B.; Cristea, I. Fuzzy Algebraic Hyperstructures-An Introduction. In Studies in Fuzziness and Soft Computing; Springer: Cham, Switzerland, 2015; Volume 321.

14. Corsini, P. A new connection between hypergroups and fuzzy sets. Southeast Asian Bull. Math. 2003, 27, 221-229.

15. Corsini, P.; Cristea, I. Fuzzy grade of i.p.s. hypergroups of order 7. Iran. J. Fuzzy Syst. 2004, 1, 15-32.

16. Cristea, I. Complete hypergroups, 1-hypergroups and fuzzy sets. An. Stiintifice Univ. Ovidius Constanta Ser. Mat. 2002, 10, 25-37.

17. Novak, M.; Cristea, I. Cyclicity in EL-hypergroups. Symmetry 2018, 10, 611. [CrossRef]

18. Antampoufis, N.; Hošková-Mayerová, Š. A brief survey on the two different approaches of fundamental equivalence relations on hyperstructures. Ratio Math. 2017, 33, 47-60.

19. Corsini, P. Prolegomena of Hypergroup Theory; Aviani Editore: Tricesimo, Italy, 1993.

20. Davvaz, B.; Hassani Sadrabadi, E.; Cristea, I. Atanassov's intuitionistic fuzzy grade of the complete hypergroups of order less than or equal to 6. Hacet. J. Math. Stat. 2015, 44, 295-315.

21. Corsini, P. Sugli ipergruppi canonici finiti con identità parziali scalari. Rend. Circ. Mat. Palermo 1987, 36, $205-219$. [CrossRef]

22. Corsini, P. (i.p.s.) Ipergruppi di ordine 6. Ann. Sc. De L'Univ. Blaise Pascal, Clermont-II 1987, $24,81-104$.

23. Corsini, P. (i.p.s.) Ipergruppi di ordine 7. Atti Sem. Mat. Fis. Univ. Modena 1986, 34, 199-216.

24. Corsini, P.; Cristea, I. Fuzzy sets and non complete 1-hypergroups. An. Stiintifice Univ. Ovidius Constanta Ser. Mat. 2005, 13, 27-54.

25. Rosenfeld, A. Fuzzy subgroups. J. Math. Anal. Appl. 1971, 35, 512-517. [CrossRef]

26. Sen, M.K.; Ameri, R.; Chowdhury, G. Fuzzy hypersemigroups. Soft Comput. 2008, 12, 891-900. [CrossRef]

27. Massouros, C.G.; Massouros, G.G. On 2-element fuzzy and mimic fuzzy hypergroups. J. AIP Conf. Proc. 2012, $1479,2213-2216$.

(C) 2020 by the authors. Licensee MDPI, Basel, Switzerland. This article is an open access article distributed under the terms and conditions of the Creative Commons Attribution (CC BY) license (http:/ / creativecommons.org/licenses/by/4.0/). 